\title{
SUFFICIENT CONDITIONS FOR THE PROBLEM OF BOLZA IN THE CALCULUS OF VARIATIONS*
}

\author{
BY \\ MAGNUS R. HESTENES $†$
}

1. Introduction. Let the end points of the arcs

$$
y_{i}=y_{i}(x) \quad\left(x^{1} \leqq x \leqq x^{2} ; i=1, \cdots, n\right)
$$

be denoted by the symbols $\left(x^{1}, y_{1}^{1}, \cdots, y_{n}{ }^{1}\right)$ and $\left(x^{2}, y_{1}{ }^{2}, \cdots, y_{n}{ }^{2}\right)$. The problem to be considered is that of finding in a class of arcs (1:1) and sets $(\alpha)=\left(\alpha_{1}, \cdots, \alpha_{r}\right)$ satisfying the differential equations and end conditions

$$
\begin{array}{cr}
\phi_{\beta}\left(x, y, y^{\prime}\right)=0 & (\beta=1, \cdots, m<n), \\
x^{s}=x^{s}(\alpha), y_{i}{ }^{s}=y_{i}{ }^{s}(\alpha) & (s=1,2)
\end{array}
$$

one which minimizes a functional of the form

$$
J=\theta(\alpha)+\int_{x^{2}}^{x^{2}} f\left(x, y, y^{\prime}\right) d x .
$$

This problem was first formulated by Bolza (II, p. 431) $\ddagger$ and will be called the problem of Bolza. The formulation here given is due to Morse and Myers (VI, p. 236). Of special importance is the case in which the end conditions $(1: 3)$ are of the form

$$
\begin{array}{ll}
x^{1}=x^{1}\left(\alpha_{1}, \cdots, \alpha_{\rho}\right), & y_{i}{ }^{1}=y_{i}{ }^{1}\left(\alpha_{1}, \cdots, \alpha_{p}\right), \\
x^{2}=x^{2}\left(\alpha_{\rho+1}, \cdots, \alpha_{r}\right), & y_{i}{ }^{2}=y_{i}{ }^{2}\left(\alpha_{p+1}, \cdots, \alpha_{r}\right)
\end{array}
$$

and the function $\theta(\alpha)$ is of the form

$$
\theta(\alpha)=\theta^{1}\left(\alpha_{1}, \cdots, \alpha_{\rho}\right)-\theta^{2}\left(\alpha_{\rho+1}, \cdots, \alpha_{r}\right) .
$$

The latter problem will be called the problem of Bolza with separated end conditions. In the proof of Theorem 9:2 below it will be shown that the two problems are equivalent not only in the sense that each can be transformed into one of the other type but also in the sense that the theory of the one can be deduced from that of the other.

* Presented to the Society, June 23, 1933, and March 31, 1934; received by the editors January $25,1934$.

† National Research Fellow. A considerable portion of the results here given were obtained while the author was a Research Assistant to Professor Bliss at the University of Chicago.

$\ddagger$ Roman numerals in parentheses refer to the list of references at the end of this paper. 
Sufficient conditions for a minimum in the problems of Bolza were first given by Morse (VIII) and later by Bliss (IX) and Hu (XIX). However, the normality assumptions, which they make, prevent these conditions from being applicable without further modification to the problem of Mayer (III; $\mathrm{XI})$, to the case in which the functions $\phi_{\beta}$ contain no derivatives, and to a number of other problems. Sufficient conditions for the problem of Mayer have been deduced by Bliss and Hestenes (XVII; XVIII) who make similar restrictive normality assumptions. In $\$ 9$ below we give for the first time sets of sufficient conditions for the problem of Bolza containing no normality assumptions whatsoever. We merely assume the existence of a set of multipliers of the form $\lambda_{0}=1, \lambda_{\beta}(x)$ with which the arc $g$ under consideration satisfies suitable analogues of the usual sufficiency conditions. It is clear that the results of the present paper are applicable at once to the problem of Mayer and thus unify the problems of Bolza and Mayer so that they are equivalent not only in the sense that each can be transformed into one of the other type but also in the sense that the theory for the one can be deduced from that of the other without further modification. The results of this paper also show that the classical problem of Mayer can be considered as a problem of Lagrange with one variable end point (cf. I, p. 224). Moreover by the use of a device given by Bliss $(\mathrm{V}$, p. 703) the results here given can be applied to the case in which the functions $\phi_{\beta}$ contain no derivatives. One obtains thereby an extension of the results given by Bower (XX).

In order to obtain the sufficient conditions here given we derive in $\$ 4$ a new analogue of the necessary condition of Mayer for the problem of Bolza with separated end conditions. A similar condition has been given by Currier (XII, p. 699) for parametric problems without differential side conditions and with special end conditions. The methods of Currier, however, do not seem to be readily extensible to the problem of Bolza without making stringent normality assumptions. A very special case of this necessary condition has been given by Bliss for variable end point problems in the plane (IV, pp. 324-6).

The sufficiency proof given in $\$ \S 6$ and 9 below is new and is simpler than those given hitherto for the problems of Bolza. It is a direct extension of the classical method used for fixed end point problems and does not make use of the famous theorem of Hahn (IX, p. 267).

The author has made extensive use of the papers of Bliss and Morse listed at the end of this paper.

2. First necessary conditions. Let us suppose that we have given an open region $\Re$ of points $\left(x, y, y^{\prime}\right)$ in which the functions $f, \phi_{\beta}$ have continuous derivatives of the first three orders. A set $\left(x, y, y^{\prime}\right)$ is said to be admissible if it is in $\Re$ and satisfies the equations $\phi_{\beta}=0$. A differentiably admissible arc is 
a continuous arc having a continuously turning tangent except possibly at a finite number of points on it and having all of its elements $\left(x, y, y^{\prime}\right)$ admissible. A differentiably admissible arc $(1: 1)$ and a set of constants $(\alpha)=\left(\alpha_{1}, \cdots, \alpha_{r}\right)$ satisfying the end conditions $(1: 3)$ are said to form an admissible arc.

We center our attention on a particular admissible arc $g$ and propose to find under what conditions $g$ will surely furnish a minimum to $J$ relative to neighboring admissible arcs. We assume that the matrix $\left\|\phi_{\beta v_{i}{ }^{\prime}}\right\|$ has rank $m$ on $g$ and that the set $(\alpha)$ belonging to $g$ is the set $(\alpha)=(0)$. The functions $\theta(\alpha), x^{s}(\alpha), y_{i}{ }^{g}(\alpha)(s=1,2)$ are assumed to have continuous first and second partial derivatives near $(\alpha)=(0)$.

The tensor analysis summation convention will be used throughout.

The following necessary condition is well known and has been established by Morse and Myers (VI, p. 245) and by Bliss and Schoenberg (X, pp. 681-3) and by others.

THEOREM 2:1. If $g$ affords a minimum to $J$ then there exist for it constants $c_{1}, \cdots, c_{n}$ and a function $F=\lambda_{0} f+\lambda_{\beta}(x) \phi_{\beta}(\beta=1, \cdots, m)$ such that the equations

$$
F_{y^{\prime}}=\int_{x^{1}}^{x} F_{y_{i}} d x+c_{i}, \quad \phi_{\beta}=0 \quad(i=1, \cdots, n)
$$

hold at every point of $g$. Moreover on $g$ the equation

$$
\left[\left(F-y_{i}^{\prime} F_{y^{\prime}}\right) d x+F_{y_{i}{ }^{\prime}} d y_{i}\right]_{1}^{2}+\lambda_{0} d \theta=0^{*}
$$

is an identity in $d \alpha_{h}$ when the differentials $d x^{1}, d y_{i}{ }^{1}, d x^{2}, d y_{i}{ }^{2}, d \theta$ are expressed in terms of the differentials $d \alpha_{h}$. The multiplier $\lambda_{0}$ is a constant. The multipliers $\lambda_{\beta}(x)$ are continuous except possibly at values of $x$ defining corners of $g$. The elements of the set $\lambda_{0}, \lambda_{\beta}(x)$ do not vanish simultaneously at any point on $g$.

By the order $q$ of anormality of $g$ on an interval $x^{\prime} x^{\prime \prime}$ relative to the conditions $(2: 1)$ is meant the number $q$ of linearly independent sets of multipliers of the form $\lambda_{0}=0, \lambda_{\beta}(x)$ with which $g$ satisfies the conditions $(2: 1)$ on $x^{\prime} x^{\prime \prime}$. The order $q$ of $g$ on $x^{\prime} x^{\prime \prime}$ cannot exceed the number $m$ of differential equations $\phi_{\beta}=0$. This follows because for every $m+1$ sets of multipliers of the form $\lambda_{0}=0, \lambda_{\beta}(x)$ there exists at least one linear combination of these sets having constant coefficients not all zero and vanishing at $x^{\prime}$ and hence vanishing for all values of $x$ on $x^{\prime} x^{\prime \prime}$. The case $q=0$ on every sub-interval of $x^{1} x^{2}$ has been

* The symbol [ $]_{1}^{2}$ denotes the value of [ ] at the final end point 2 on $g$ minus its value at the initial end point 1 on $g$. 
treated by Morse and Bliss. In this case $g$ is said to be normal on every subinterval.

Carathéodory (XV, XVI) has shown that in the analytic case the order $q$ of anormality of $g$ is the same on every sub-interval of $x^{1} x^{2}$. In the nonanalytic case this is not necessarily true, as will be seen in the example given at the end of $\$$.

By the order.p of anormality of $g$ relative to the conditions $(2: 1)$ and $(2: 2)$ is meant the number $p$ of linearly independent sets of multipliers of the form $\lambda_{0}=0, \lambda_{\beta}(x)$ with which $g$ satisfies the conditions $(2: 1)$ and $(2: 2)$. Clearly the order $p$ of $g$ cannot exceed the order $q$ of $g$ on the interval $x^{1} x^{2}$ defined by its end points. If $p=0$ then $g$ is said to be normal. In the normal case there exists an infinity of admissible arcs in every neighborhood of $g$. In the anormal case this is not necessarily true. Moreover for a normal minimizing arc $g$ there exists a unique set of multipliers of the form $\lambda_{0}=1, \lambda_{\beta}(x)$ satisfying the conditions of Theorem 2:1 (V, pp. 693-5).

We have the following analogue of the necessary condition of Weierstrass which has been established by Graves (XIII, p. 751).

THEOREM 2:2. If $g$ is a normal minimizing arc then at each element $(x, y$, $\left.y^{\prime}, \lambda\right)$ on $g$ the inequality

$$
E\left(x, y, y^{\prime}, \lambda, Y^{\prime}\right) \geqq 0
$$

must hold for every admissible set $\left(x, y, Y^{\prime}\right) \neq\left(x, y, y^{\prime}\right)$ whose matrix $\left\|\phi_{\beta y_{i^{\prime}}}\left(x, y, Y^{\prime}\right)\right\|$ has rank $m$, where

$$
\begin{aligned}
E\left(x, y, y^{\prime}, \lambda, Y^{\prime}\right)=F(x, y, & \left.Y^{\prime}, \lambda\right) \\
& -F\left(x, y, y^{\prime}, \lambda\right)-\left(Y_{i}^{\prime}-y_{i}^{\prime}\right) F_{y^{\prime}}\left(x, y, y^{\prime}, \lambda\right) .
\end{aligned}
$$

The analogue of the necessary condition of Clebsch given in Theorem 4:5 below can also be obtained from Theorem $2: 2$ by the arguments given by Bliss (V, pp. 718-9).

An extremal arc is defined to be a differentiably admissible arc and a set of multipliers

$$
y_{i}=y_{i}(x), \lambda_{\beta}=\lambda_{\beta}(x) \quad\left(x^{1} \leqq x \leqq x^{2}\right)
$$

having continuous derivatives $y_{i}^{\prime}, y_{i}^{\prime \prime}, \lambda_{\beta}^{\prime}$ and satisfying with $\lambda_{0}=1$ the Euler-Lagrange equations

$$
(d / d x) F_{y_{i}^{\prime}}-F_{y_{i}}=0, \phi_{\beta}=0 .
$$

An extremal is said to be non-singular if the determinant

$$
\left|\begin{array}{ll}
F_{y_{i}^{\prime} y_{k^{\prime}}} & \phi_{\beta y_{i^{\prime}}} \\
\phi_{\beta y_{k^{\prime}}} & 0
\end{array}\right|
$$


is different from zero at each element $\left(x, y, y^{\prime}, \lambda\right)$ on it. A study of the extremal family has been made by Bliss (V, p. 687).

In the sequel it will be understood that the admissible arc $g$ under consideration is an extremal arc satisfying the conditions (2:1) and (2:2) of Theorem 2:1 unless otherwise expressly stated.

3. The second variation and the accessory minimum problem. In this section we are concerned with the functional

$$
J_{2}(\eta, w)=b_{h l} w_{h} w_{l}+\int_{x^{1}}^{x^{2}} 2 \omega\left(x, \eta, \eta^{\prime}\right) d x \quad(h, l=1, \cdots, r)
$$

evaluated along the extremal $g$, where ( $s$ not summed; $s=1,2$ )

$$
\begin{aligned}
& b_{h l}=\theta_{h l}+\mathrm{l}\left(F_{x}-y_{i}^{\prime} F_{y_{i}}\right) x_{h}^{s} x_{l}^{*}+\left(F-y_{i}^{\prime} F_{y_{i^{\prime}}}\right) x_{h l}^{\prime} \\
& \left.+F_{y_{i}}\left(x_{h}^{s} y_{i l}^{s}+x_{i}^{s} y_{i h}^{s}\right)+F_{y_{i}^{\prime}} y_{i h l}^{*}\right]_{1}^{2} \text {, }
\end{aligned}
$$

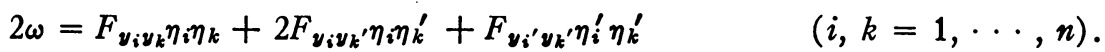

Here the symbols $x^{s}, y_{i}$ denote the functions $x^{s}(\alpha), y_{i}{ }^{s}(\alpha)$ and the subscripts $h$ and $l$ denote differentiation with respect to $\alpha_{h}$ and $\alpha_{l}$ respectively at $(\alpha)=(0)$. The matrix $\left\|b_{l l l}\right\|$ is symmetric. The functions $\eta_{i}(x)$ are assumed to possess continuous derivatives except possibly at a finite number of values of $x$ on the interval $x^{1} x^{2}$ and to satisfy with the constants $w_{h}$ the equations

$$
\begin{aligned}
& \Phi_{\beta}\left(x, \eta, \eta^{\prime}\right)=\phi_{\beta y_{i}} \eta_{i}+\phi_{\beta y_{i}} \eta^{\prime} !=0, \\
& \eta_{i}^{s}=\stackrel{s}{c}_{i h} w_{h} \quad(s=1,2 ; h=1, \cdots, r)
\end{aligned}
$$

evaluated along $g$, where $c_{i h^{s}}=y_{i h^{s}}(0)-y_{i}^{\prime}\left(x^{s}\right) x_{h}^{s}(0)$ (s not summed). Such a set $\eta_{i}, w_{h}$ is called a set of admissible variations for $g$. The functional $J_{2}(\eta, w)$ is called the second variation of the functional $J$ along $g$ (cf. VIII, pp. 520-1).

ThEOREM 3:1. If $g$ is a normal minimizing extremal arc, then along $g$ the second variation $J_{2}$ of $J$ must satisfy the condition $J_{2}(\eta, w) \geqq 0$ for every set of admissible variations $\eta_{i}, w_{h}$ having continuous second derivatives except possibly at a finite number of values of $x$ on the interval $x^{1} x^{2}$ defined by the end points of $g$.

The theorem follows readily from the derivation of the second variation given by Morse (VIII, pp. 520-1) provided that we show that for every set of admissible variations $\eta_{i}, w_{h}$ having the continuity properties described in. the theorem there exists a one-parameter family of admissible arcs

$$
y_{i}=y_{i}(x, e), \quad \alpha_{h}=\alpha_{h}(e) \quad\left[x^{1}(\alpha) \leqq x \leqq x^{2}(\alpha)\right]
$$

containing $g$ for $e=0$, having $\eta_{i}, w_{h}$ as its variations along $g$, and having the following continuity properties. The functions $y_{i}(x, e), \alpha_{h}(e)$ have continuous 
first and second derivatives with respect to $e$ near $e=0$. The derivatives $y_{i x e}, y_{i x e e}, y_{i x x}$ exist and are continuous for values $(x, e)$ near those belonging to $g$ except possibly at a finite number of values of $x$ on $x^{1} x^{2}$. The existence of such a family is readily established by the methods of Bliss (V, p. 695: cf. VI, p. 249) with suitable modifications in order to obtain the necessary derivatives.

Theorem 3:1 leads us to the study of the accessory minimum problem, namely, the problem of minimizing the functional $J_{2}(\eta, w)$ in the class of admissible variations $\eta_{i}, w_{h}$. This problem is a problem of Bolza of the type described in $\$ 1$. From Theorem 2:1 we obtain the following equations which a minimizing arc without corners must satisfy:

$$
\begin{array}{cr}
(d / d x) \Omega_{\eta^{i^{\prime}}}-\Omega_{\eta_{i}}=0, \Phi_{\beta}=0 & (\beta=1, \cdots, m), \\
\eta_{i}^{s}-c_{i h}^{s} w_{h}=0 \quad(i=1, \cdots, n ; s=1,2), \\
\zeta_{i}^{2} c_{i h}^{2}-\zeta_{i}^{1} c_{i h}^{1}+\mu_{0} b_{h l} w_{l}=0 & (h, l=1, \cdots, r),
\end{array}
$$

where $\Omega=\mu_{0} \omega+\mu_{\beta}(x) \Phi_{\beta}, \zeta_{i}=\Omega_{\eta_{i^{\prime}}}$. The equations (3:1) are known as the $a c$ cessory equations, the equations $(3: 2)$ as the secondary end conditions, the equations $(3: 3)$ as the secondary transversality conditions. The extremals for this problem will be called secondary extremals. The secondary end conditions are said to be regular in case the $2 n \times r$-dimensional matrix $\left\|c_{i h^{\prime}}\right\|$ has rank $r$ on $g$.

If $g$ is non-singular the equations

$$
\zeta_{i}=\Omega_{\eta_{i^{\prime}}}\left(x, \eta, \eta^{\prime}, \mu\right), \quad \Phi_{\beta}\left(x, \eta, \eta^{\prime}\right)=0
$$

with $\mu_{0}=1$ can be solved for the variables $\eta_{i}^{\prime}, \mu_{\beta}$. The accessory equations with $\mu_{0}=1$ are then found to be equivalent to equations of the form

$$
d \eta_{i} / d x=G_{i}(x, \eta, \zeta), \quad d \zeta_{i} / d x=H_{i}(x, \eta, \zeta),
$$

where $G_{i}, B_{i}$ are linear in the variables $\eta_{i}, \zeta_{i}(\mathrm{~V}$, p. 727). For every pair of solutions $\eta_{i}, \zeta_{i}$ and $u_{i}, v_{i}$ of these equations the expression $\zeta_{i} u_{i}-\eta_{i} v_{i}$ is a constant $(\mathrm{V}, \mathrm{p} .738)$. If this constant is zero the solutions are said to be conjugate solutions. A set of $n$ mutually conjugate linearly independent solutions is said to form a conjugate system.

In the separated end point case the quadratic form $b_{h l} w_{h} w_{l}$ is of the form

$$
b_{\mu \nu}^{1} w_{\mu} w_{\nu}-b_{\sigma \tau}^{2} w_{\sigma} w_{\tau} \quad(\mu, \nu=1, \cdots, \rho ; \sigma, \tau=\rho+1, \cdots, r),
$$

where

$$
\begin{aligned}
b_{\mu \nu}^{1}= & \theta_{\mu \nu}^{1}-\left(F_{x}-y_{i}^{\prime} F_{y_{i}}\right) x_{\mu}^{1} x_{\nu}^{1}-\left(F-y_{i}^{\prime} F_{y^{\prime}}\right) x_{\mu \nu}^{1} \\
& -F_{y_{i}}\left(x_{\mu}^{1} y_{i \nu}^{1}+x_{\nu} y_{i \mu}^{1}\right)-F_{y^{\prime}} y_{i \mu \nu}^{1}
\end{aligned}
$$


evaluated at the initial point 1 on $g$ and $b_{\sigma \tau}{ }^{2}$ is a similar expression in $\theta_{\sigma \tau}{ }^{2}$, $x_{\sigma}^{2}, y_{i \sigma}{ }^{2}, x_{\sigma \tau}{ }^{2}, y_{i \sigma \tau}{ }^{2}$ evaluated at the final end point 2 on $g$. The matrices $\left\|b_{\mu \nu}{ }^{1}\right\|$ and $\left\|b_{\sigma \tau}{ }^{2}\right\|$ are symmetric. Moreover the equations (3:2) and (3:3) with $\mu_{0}=1$ can be written in the form

$$
\begin{aligned}
\eta_{i}^{1} & =\stackrel{1}{c_{i \mu} w_{\mu},} \zeta_{i}^{1} c_{i \mu}^{1}=b_{\mu \nu}^{1} w_{\nu} & (\mu, \nu=1, \cdots, \rho), \\
\eta_{i}^{2} & =c_{i \sigma}^{2} w_{\sigma}, \quad \zeta_{i}^{2} c_{i \sigma}^{2}=b_{\sigma \tau}^{2} w_{\tau} & (\sigma, \tau=\rho+1, \cdots, r) .
\end{aligned}
$$

If the matrix $\left\|c_{i \mu}{ }^{1}\right\|$ has rank $\rho$ then there are $n$ and at most $n$ linearly independent solutions

$$
\eta_{i k}(x), \zeta_{i k}(x), w_{\mu k} \quad(k=1, \cdots, n)
$$

of equations $(3: 4)$ and $(3: 6)$, as one readily verifies. Moreover the secondary extremals $\eta_{i k}, \zeta_{i k}$ in (3:8) form a conjugate system since at $x=x^{1}$ we have

$$
\begin{aligned}
\zeta_{i k} \eta_{i j}-\zeta_{i j} \eta_{i k} & =\zeta_{i k} c_{i \mu}^{1} w_{\mu j}-\zeta_{i j} c_{i \mu}^{1} w_{\mu k} \\
& =b_{\mu \nu}^{1} w_{\mu j} w_{\nu k}-b_{\mu \nu}^{1} w_{\mu k} w_{\nu j}=0
\end{aligned}
$$

and since these secondary extremals are linearly independent, as follows readily from the fact that the matrix $\left\|c_{i \mu}{ }^{1}\right\|$ has rank $\rho$. Similarly if the matrix $\left\|c_{i \sigma}{ }^{2}\right\|$ has rank $r-\rho$, then there are $n$ and at most $n$ linearly independent solutions

$$
u_{i k}(x), v_{i k}(x), w_{\sigma k} \quad(k=1, \cdots, n)
$$

of equations (3:4) and (3:7). It is clear that the secondary extremals $u_{i k}, v_{i k}$ also form a conjugate system.

The following lemma will be useful:

LEMMA 3:1. The order $p$ of anormality of $g$ is equal to the number of linearly independent secondary extremals $\eta_{i}, \mu_{\beta}$ having $(\eta) \equiv(0)$ on $x^{1} x^{2}$ and satisfying the equations $(3: 3)$ with the set $(w)=(0)$.

This result follows because the first $n$ equations (2:4) with $\lambda_{0}=0$ are equivalent to the first $n$ equations $(3: 1)$ with $(\eta) \equiv(0)$. Moreover the transversality condition $(2: 2)$ with $\lambda_{0}=0$ is equivalent to the conditions $(3: 3)$ with $(\eta) \equiv(0)$ and $(w)=(0)$.

Similarly we have

LEMMA 3:2. The order $q$ of anormality of $g$ on the interval $x^{1} x^{2}$ is equal to the number of linearly independent secondary extremals $\eta_{i}, \mu_{\beta}$ having $(\eta) \equiv(0)$ on $x^{1} x^{2}$.

A further lemma is the following: 
LEMMA 3:3. If $u_{i}, v_{i}$ is a secondary extremal having $(u) \equiv(0)$ on $x^{1} x^{2}$ then the relation $v_{i} \eta_{i}=$ constant holds for every differentiably admissible arc $\eta_{i}(x)$ for the accessory minimum problem.

Let $\mu_{\beta}=\lambda_{\beta}(x)$ be the multipliers belonging to the secondary extremal $u_{i}, v_{i}$. The lemma now follows readily by multiplying the equations of variation

$$
\phi_{\beta y_{i}} \eta_{i}+\phi_{\beta y_{i}} \eta_{i}^{\prime}=0
$$

by the functions $\lambda_{\beta}(x)$, adding, and applying the usual integration by parts with the help of equations $(2: 4)$ with $\lambda_{0}=0$.

An important consequence of Lemma 3:3 is that the accessory minimum problem can be modified so that its admissible arcs are all normal. This can be done by replacing the secondary end conditions $(3: 2)$ by the conditions

$$
\stackrel{1}{\eta_{i}}=\stackrel{1}{c_{i h}} w_{h}, \quad \eta_{i}^{2}=\stackrel{2}{c_{i h}} w_{h}+\zeta_{i \gamma}^{2} w_{r+\gamma} \quad(\gamma=1, \cdots, p)
$$

where $p$ is the order of anormality of $g$ and $\eta_{i \gamma}, \zeta_{i \gamma}$ are $p$ linearly independent secondary extremals having the properties described in Lemma 3:1. We may suppose that these secondary extremals have been chosen so that the columns of the matrix $\left\|\zeta_{i \gamma}{ }^{2}\right\|$ are normed and orthogonalized. By Lemma 3:1 we have

$$
\zeta_{i \gamma}^{2} c_{i h}^{2}-\zeta_{i \gamma}^{1} c_{i h}^{1}=0 \quad(\gamma=1, \cdots, p) .
$$

Multiplying the equations $(3: 10)$ by the values $-\zeta_{i \gamma}{ }^{1}, \zeta_{i \gamma}{ }^{2}$ and adding, it is found with the help of equations (3:11) and Lemma $3: 3$ that the equations

$$
0=-\zeta_{i \gamma}^{1} \eta_{i}^{1}+\zeta_{i \eta}^{2} \eta_{i}^{2}=w_{r+\gamma} \quad(\gamma=1, \cdots, p)
$$

hold for every admissible arc $\eta_{i}, w_{h}, w_{r+\gamma}$ for the new problem. The new problem is therefore equivalent to the original one. Moreover every admissible arc for the new problem is normal, by Lemma $3: 1$, since the secondary extremals $\eta_{i \gamma}, \zeta_{i \gamma}$, described above, do not satisfy the analogue of conditions (3:3) with the set $w_{h}=w_{r+\gamma}=0$.

LEMMA 3:4. If $g$ is non-singular then a minimizing arc $g_{2}$ for the accessory minimum problem must be an arc defined by a secondary extremal and hence can have no corners.

As was seen above we may assume that $g_{2}$ is normal. Since $g_{2}$ is a minimizing arc there exists for it a unique function $\Omega=\omega+\mu_{\beta} \Phi_{\beta}$ with which $g_{2}$ satisfies the conditions implied by Theorem 2:1. The functions $\zeta_{i}=\Omega_{\eta_{i^{\prime}}}$ are therefore continuous along $g_{2}$. The non-singularity of $g$ and hence of $g_{2}$ implies that the functions $\eta_{i}, \mu_{\beta}$ belonging to $g_{2}$ define extremal segments between corners of $g_{2}(\mathrm{~V}, \mathrm{p} .684)$. The continuity of the functions $\zeta_{i}$ now implies that 
the arc $g_{2}$ can have no corners since there is one and only one secondary extremal taking given values $\eta_{i}{ }^{0}, \zeta_{i}{ }^{0}$ at a value $x=x^{0}$ on $x^{1} x^{2}$. This proves the lemma.

4. Necessary conditions for the second variation to be positive. The second variation $J_{2}(\eta, w)$ is said to be positive along $g$ if the inequality $J_{2}(\eta, w) \geqq 0$ holds for every set of admissible variations $\eta_{i}, w_{h}$ belonging to $g$. The results of this section will remain valid if we further restrict these variations to have the continuity properties described in Theorem $3: 1$. The necessary conditions here given must therefore be satisfied if $g$ is to be a normal minimizing arc for the original problem.

We have the following necessary condition in the separated end point case. The relations between this condition and those of Currier and Bliss have been explained in $\$ 1$.

THEOREM 4:1. If in the separated end point case the extremal $g$ is nonsingular, the secondary end conditions are regular, and the second variation $\mathrm{J}_{2}$ is positive along $g$, then at each point $x^{3}$ on $x^{1} x^{2}$ the inequality

$$
\left(\zeta_{i j} u_{i k}-\eta_{i j} v_{i k}\right) a_{j} b_{k} \geqq 0 \quad(i, j, k=1, \cdots, n)
$$

must hold for every set of constants $\left(a_{j}, b_{k}\right)$ satisfying the equations

$$
\eta_{i j}\left(x^{3}\right) a_{j}=u_{i k}\left(x^{3}\right) b_{k}
$$

where $\eta_{i j}, \zeta_{i j}$ and $u_{i k}, v_{i k}$ are the conjugate systems belonging to the sets (3:8) and (3:9) respectively. The coefficients in the bilinear form (4:1) are constants.

In order to prove the theorem we note that a set of constants $\left(a_{j}, b_{k}\right)$ satisfying the equations $(4: 2)$ determines a broken secondary extremal $\eta_{i}, \zeta_{i}$ defined by the equations

$$
\begin{aligned}
& \eta_{i}=\eta_{i j} a_{j}, \zeta_{i}=\zeta_{i j} a_{j} \text { on } x^{1} \leqq x \leqq x^{3}, \\
& \eta_{i}=u_{i k} b_{k}, \zeta_{i}=v_{i k} b_{k} \text { on } x^{3} \leqq x \leqq x^{2}
\end{aligned}
$$

and satisfying the conditions $(3: 6)$ and $(3: 7)$ with the set of constants $w_{\mu}=w_{\mu j} a_{j}, w_{\sigma}=w_{\sigma k} b_{k}$. Let $\mu_{\beta}(x)$ be the set of multipliers belonging to the broken extremal $\eta_{i}, \zeta_{i}$. With the help of the formula

$$
2 \Omega=\eta_{i} \Omega_{\eta_{i}}+\eta_{i}^{\prime} \Omega_{\eta_{i}}+\mu_{\beta} \Omega_{\mu \beta}
$$

and the usual integration by parts it is found that along this broken extremal the second variation $J_{2}$ is expressible in the form

$$
\begin{aligned}
J_{2} & =b_{\mu \nu}^{1} w_{\mu} w_{\nu}-b_{\sigma \tau}^{2} w_{\sigma} w_{\tau}+\int_{x^{1}}^{x^{3}} 2 \Omega d x+\int_{x^{3}}^{x^{2}} 2 \Omega d x \\
& =b_{\mu \nu}^{1} w_{\mu} w_{\nu}-b_{\sigma \tau}^{2} w_{\sigma} w_{\tau}+\left[\eta_{i} \zeta_{i}\right]_{1}^{2}+\left[\eta_{i} \zeta_{i}\right]_{x^{3}+0}^{x^{3}-0} .
\end{aligned}
$$


By the use of equations (3:6), (3:7), (4:2), and (4:3) it follows readily that

$$
\begin{aligned}
J_{2} & =\eta_{i}\left(x^{3}+0\right) \zeta_{i}\left(x^{3}-0\right)-\zeta_{i}\left(x^{3}+0\right) \eta_{i}\left(x^{3}-0\right) \\
& =\left(\zeta_{i j} u_{i k}-\eta_{i j} v_{i k}\right) a_{j} b_{k} .
\end{aligned}
$$

This formula justifies the inequality $(4: 1)$. The last statement in the theorem follows from the remarks made in the paragraph containing the equations (3:4). The theorem is now proved.

Consider now the problem of Bolza in which the end conditions are not necessarily separated. Suppose for the moment that $g$ is non-singular. Let $\eta_{i \rho}, \mu_{\beta_{\rho}}, w_{h_{\rho}}(\rho=1, \cdots, \nu)$ be a maximum set of linearly independent secondary extremals and constants $(w)$ satisfying the secondary end conditions $(3: 2)$. It is clear that the quadratic form

$$
Q(z)=J_{2}\left(\eta_{\rho} z_{\rho}, w_{\rho} z_{\rho}\right)
$$

in the constants $\left(z_{1}, \cdots, z_{n}\right)$ must be positive on $g$ if the second variation $J_{2}$ is to be positive along $g$. This proves the first part of the following theorem:

THEOREM 4:2. If the extremal $g$ is non-singular and the second variation $J_{2}$ is positive along $g$, then the quadratic form (4:5) must be positive on $g$. Moreover at each point $x^{3}$ on $x^{1} x^{2}$ the inequality (4:1) must hold for every set of constants $\left(a_{j}, b_{k}\right)$ satisfying the equations $(4: 2)$, where $\eta_{i j}, \zeta_{i j}$ and $u_{i k}$, $v_{i k}$ are conjugate systems of secondary extremals having $\eta_{i j}\left(x^{1}\right)=u_{i k}\left(x^{2}\right)=0$.

The last part of the theorem is obtained by applying Theorem $4: 1$ to the case in which the secondary end conditions are of the form $\eta_{i}{ }^{1}=0, \eta_{i}{ }^{2}=0$.

A value $x^{3} \neq x^{1}$ is said to define a point 3 conjugate to 1 on $g$ if there exists a secondary extremal $\eta_{i}=u_{i}(x), \mu_{\beta}=\mu_{\beta}(x)$ having $u_{i}\left(x^{1}\right)=u_{i}\left(x^{3}\right)=0$ but not $(u) \equiv(0)$ on $x^{1} x^{3}$.

The following necessary condition is a direct extension of a condition given by Bliss (IX, p. 266).

TheOREM 4:3. If the extremal $g$ is non-singular and the second variation $\mathrm{J}_{2}$ is positive along $g$, then the quadratic form (4:5) must be positive on $g$. Moreover there can be no point 3 conjugate to 1 on $g$ between its end points 1 and 2 defined by a secondary extremal $u_{i}(x), \rho_{\beta}(x)$ with $\left(u^{\prime}\right) \neq(0)$ at $x=x^{3}$. If the order $q$ of anormality of $g$ is the same on every sub-interval $x^{3} x^{2}$ of $x^{1} x^{2}$, then there can be no point 3 conjugate to 1 on $g$ between 1 and 2 .

For if there were a point 3 conjugate to 1 on $g$ between 1 and 2 defined by a secondary extremal $u_{i}, \rho_{\beta}$, then along the arc

$$
\eta_{i} \equiv u_{i}(x) \quad\left(x^{1} \leqq x \leqq x^{3}\right), \quad \eta_{i} \equiv 0 \quad\left(x^{3} \leqq x \leqq x^{2}\right), \quad w_{h}=0
$$


the second variation would take the value zero $(\mathrm{V}, \mathrm{p} .726)$. This arc would therefore be a minimizing arc for the accessory minimum problem and hence could have no corners, by Lemma 3:4. This proves the first statement concerning conjugate points.

In order to prove the last statement of the theorem we note that according to Lemma $3: 4$ the functions $\eta_{i}$ just defined would belong to a secondary extremal $\eta_{i}, \mu_{\beta}$. The functions $\eta_{i}$ would then be identically zero on $x^{1} x^{2}$ since, as one easily sees, Lemma $3: 2$ and our assumption concerning anormality imply that a secondary extremal $\eta_{i}, \mu_{\beta}$ having $\eta_{i} \equiv 0$ on $x^{3} x^{2}$ has $\eta_{i} \equiv 0$ on the whole interval $x^{1} x^{2}$. It follows that in this case there can be no point 3 on $g$ conjugate to 1 between 1 and 2. This completes the proof of the theorem.

By the accessory boundary value problem is meant the equations

$$
\begin{aligned}
& (d / d x) \Omega_{\eta_{i^{\prime}}}-\Omega_{\eta_{i}}+\sigma \eta_{i}=0, \Phi_{\beta}=0, \\
& \eta_{i}^{*}=\stackrel{\bullet}{c_{i h} w_{h}, \zeta_{i}^{2} c_{i h}^{1}-\zeta_{i}^{1} c_{i h}^{1}+b_{h l} w_{l}=0} \quad(s=1,2),
\end{aligned}
$$

where $\Omega=\omega+\mu_{\beta} \Phi_{\beta}$. A set of functions $\eta_{i}(x), \mu_{\beta}(x)$ having continuous derivatives $\eta_{i}^{\prime}, \eta_{i}^{\prime \prime}, \eta_{\beta}^{\prime}$ and having $(\eta) \not \equiv(0)$ on $x^{1} x^{2}$ is said to form a characteristic solution if it satisfies the equations $(4: 6)$ with a set of constants $w_{h}, \sigma$. The corresponding value $\sigma$ is called a characteristic root.

We now have the further necessary condition:

Theorem 4:4. If the second variation $J_{2}(\eta, w)$ is positive along the extremal $g$ then there can be no negative characteristic roots of the accessory boundary value problem.

The proof of this theorem is well known (VIII, p. 524).

We also have the further necessary condition which is an analogue of the necessary condition of Clebsch:

THEOREM 4:5. If the second variation $J_{2}(\eta, w)$ is positive along the extremal $g$, then at each element $\left(x, y, y^{\prime}, \lambda\right)$ on $g$ the inequality

$$
F_{\boldsymbol{y}^{\prime} \boldsymbol{y}_{k^{\prime}}} \boldsymbol{\pi}_{\boldsymbol{i}} \boldsymbol{\pi}_{\boldsymbol{k}} \geqq 0
$$

must hold for every set $(\pi) \neq(0)$ which is a solution of the equations $\phi_{\beta_{y_{i}}} \pi_{i}=0$. If $g$ is non-singular then the condition (4:7) holds with the equality sign excluded.

According to the remarks preceding Lemma 3:4 we may suppose that $g$ is normal. The first statement of the theorem can now be obtained by applying Theorem 2:2 to the accessory minimum problem and by the use of Taylor's expansion. The last statement follows readily from well known theorems on quadratic forms. 
5. Criteria for conjugate points. A first criterion for conjugate points is the following one:

THEOREM 5:1. If the extremal $g$ is non-singular and if the functions $u_{i j}, v_{i j}(j=1, \cdots, 2 n)$ form $2 n$ linearly independent secondary extremals for $g$, then a value $x^{3} \neq x^{1}$ defines a point 3 conjugate to 1 on $g$ if and only if the matrix

$$
\left\|\begin{array}{c}
u_{i j}\left(x^{3}\right) \\
u_{i j}\left(x^{1}\right)
\end{array}\right\| \quad(i=1, \cdots, n ; j=1, \cdots, 2 n)
$$

has rank less than $2 n-q$, where $q$ is the order of anormality of $g$ on the interval $x^{1} x^{3}$.

The proof of this theorem can be made by the usual methods (V, p. 728) with the help of Corollary $3: 2$.

THEOREM 5:2. If the extremal $g$ is non-singular and the order $q$ of anormality of $g$ is the same on every sub-interval $x^{1} x^{3}$ of $x^{1} x^{2}$, then there exists for $g$ a conjugate system $\eta_{i k}$, $\zeta_{i k}$ of secondary extremals such that the points 3 conjugate to 1 on $g$ are determined by the zeros $x^{3} \neq x^{1}$ of the determinant $\left|\eta_{i k}\right|$.

If $q=0$ then it suffices to choose the secondary extremals $\eta_{i k}, \zeta_{i k}$ which take the initial values $\eta_{i k}\left(x^{1}\right)=0, \zeta_{i k}\left(x^{1}\right)=\delta_{i k}$, where $\delta_{i k}$ is the Kronecker delta. This follows readily from Theorem $5: 1$ by choosing the first $n$ secondary extremals of the set $u_{i j}, v_{i j}$ to be the set $\eta_{i k}, \zeta_{i k}$.

If $q>0$ we choose the first $n$ secondary extremals of the set $u_{i j}, v_{i j}$ of Theorem 5:1 such that $u_{i k}\left(x^{1}\right)=0(k=1, \cdots, n), u_{i \gamma}(x) \equiv 0(\gamma=1, \cdots, q)$ on $x^{1} x^{2}$, and such that the columns of the matrix $\left\|v_{i k}\left(x^{1}\right)\right\|$ are normed and orthogonalized. The second $n$ secondary extremals of this set are chosen so as to take the initial values $u_{i, n+k}\left(x^{1}\right)=v_{i k}\left(x^{1}\right), v_{i, n+k}\left(x^{1}\right)=0$. The secondary extremals $\eta_{i k} \equiv u_{i, q+k}, \zeta_{i k} \equiv v_{i, q+k}$ can now be shown to have the properties described in the theorem. An examination of their values at $x=x^{1}$ will show that they are mutually conjugate. Moreover it is clear that the matrix (5:1) has rank $2 n-q$ if and only if the matrix $\left\|\eta_{i \tau}\right\|=\left\|u_{i, q+\tau}\right\|(\tau=1, \cdots, n-q)$ has rank $n-q$ at $x=x^{3}$. The theorem will now follow from Theorem 5:1 if we show that the determinant $\left|\eta_{i k}\right|$ is different from zero if and only if the matrix $\left\|\eta_{i \tau}\right\|$ has rank $n-q$. If the determinant $\left|\eta_{i k}\right|$ vanishes at a value $x=x^{3}$ then there exist constants $a_{k}$ not all zero such that the equations $\eta_{i k}\left(x^{8}\right) a_{k}=0$ hold. By the use of Lemma 3:3 and by a consideration of the initial values of the secondary extremals under consideration it is found that

$$
0=v_{i \gamma}\left(x^{3}\right) \eta_{i k}\left(x^{3}\right) a_{k}=v_{i \gamma}\left(x^{1}\right) \eta_{i k}\left(x^{1}\right) a_{k}=a_{n-q+\gamma} \quad(\gamma=1, \cdots, q) .
$$

The matrix $\left\|\eta_{i \tau}\right\|(\tau=1, \cdots, n-q)$ must therefore have rank less than $n-q$ 
whenever the determinant $\left|\eta_{i k}\right|$ vanishes. The converse is immediate, and the theorem is established.

6. A fundamental sufficiency theorem. The notion of a Mayer field $\mathfrak{F}$ used here is that given by Bliss (V, p. 730). The slope functions and the multipliers belonging to $\mathfrak{F}$ will be denoted by the symbols $p_{i}(x, y), \lambda_{\beta}(x, y)$. The Hilbert integral

$$
I^{*}=\int\left\{F(x, y, p, \lambda) d x+\left(d y_{i}-p_{i} d x\right) F_{y^{\prime}}(x, y, p, \lambda)\right\}
$$

formed for these functions and $\lambda_{0}=1$ is independent of the path in $\mathfrak{F}$. The value of the integral $I^{*}$ along an extremal of the field is equal to that of the integral

$$
I=\int f\left(x, y, y^{\prime}\right) d x .
$$

The Weierstrass $E$-function $E\left(x, y, p, \lambda, y^{\prime}\right)$ is the expression (2:3).

If $g$ is an extremal of a Mayer field then the transversality condition (2:2) for $g$ implies that the equation

$$
\left[d I^{*}\right]_{1}^{2}+d \theta=0
$$

is an identity in $d \alpha_{h}$ on $g$ when the differentials $d x^{1}, d y_{i}^{1}, d x^{2}, d y_{i}^{2}, d \theta$ are expressed in terms of the differentials $d \alpha_{h}$. It follows readily that on $g$ the second differential

$$
\left[d^{2} I^{*}\right]_{1}^{2}+d^{2} \theta
$$

is a quadratic form in the variables $d \alpha_{h}$. With this in mind we can prove the following theorem:

Theorem 6:1. Let F be a Mayer field in which the inequality

$$
E\left[x, y, p(x, y), \lambda(x, y), y^{\prime}\right]>0
$$

holds for every admissible set $\left(x, y, y^{\prime}\right) \neq(x, y, p)$. If $g$ is an extremal of the field such that the equation $(6: 2)$ is an identity in $d \alpha_{h}$ on $g$ and such that the quadratic form (6:3) is positive definite on $g$, then $g$ affords a proper minimum to $J$ relative to admissible arcs $C$ in $₹$ with sets $(\alpha)$ near $(0)$.

Let $A^{1}, A^{2}$ be the arcs in $\mathfrak{F}$ defined by the equations

$$
x^{*}=x^{*}(t \alpha), \quad y_{i}^{*}=y_{i}^{*}(t \alpha) \quad(0 \leqq t \leqq 1 ; s=1,2)
$$

for a set $(\alpha)$ near $(0)$, where the functions on the right are those appearing in equations $(1: 3)$. The condition $(6: 2)$ and the positive definiteness of the 
quadratic form (6:3) tell us that the set $(\alpha)=(0)$ furnishes a proper minimum to the function

$$
W(\alpha)=\theta(\alpha)-\theta(0)+I^{*}\left(A^{2}\right)-I^{*}\left(A^{1}\right)
$$

relative to sets $(\alpha)$ near $(0)$.

Suppose now that the set $(\alpha)$ belongs to an admissible arc $C$ in $\mathfrak{F}$. With the help of the formula $(2: 3)$ and the invariant property of the integral $I^{*}$ it is found that

$$
\begin{aligned}
I(C)-I(g) & =\int_{C} E d x+I^{*}(C)-I^{*}(g) \\
& =\int_{C} E d x+I^{*}\left(A^{2}\right)-I^{*}\left(A^{1}\right),
\end{aligned}
$$

where $I$ is the integral $(6: 1)$. When the expression $\theta(\alpha)-\theta(0)$ is added to both sides of the last equation, the formula

$$
J(C)-J(g)=\int_{C} E d x+W(\alpha)
$$

is obtained. Hence we have $J(C) \geqq J(g)$ provided that the set $(\alpha)$ belonging to the arc $C$ is near (0). The equality holds only in case $(\alpha)=(0)$ and the integral of the $E$-function vanishes, that is, only in case the ends of $C$ coincide with those of $g$ and the equations $y_{i}^{\prime}-p_{i}=0$ hold along $C$. The $\operatorname{arc} C$ would then be an extremal of the field and would coincide with $g$ since there is but one extremal of the field through each point of $\mathfrak{F}$ (cf. V, pp. 731-2).

In the sequel we shall apply Theorem 6:1 only to the problem of Bolza with separated end points. If the end conditions are not of the form $(1: 4)$ then it is not always possible to construct a field such that the quadratic form (6:3) is positive definite on $g$. This can be seen by considering the special problem in $\left(x y_{1} y_{2}\right)$-space for which $\theta=\alpha, f \equiv 0, x^{1}(\alpha)=0, y_{1}^{1}(\alpha)=0, y_{2}^{1}(\alpha)=-\alpha$, $x^{2}(\alpha)=1, y_{1}^{2}(\alpha)=0, y_{2}^{2}(\alpha)=\alpha$, and $\phi_{1}=\left(1+y_{1}^{\prime 2}\right)^{1 / 2}-y_{2}^{\prime}=0$. The sufficient conditions given in $\$ 9$ below, however, are applicable to this problem.

7. Three lemmas. Consider first the problem of Bolza with separated end conditions. Suppose that $g$ is non-singular and that the secondary end conditions are regular on $g$. The arc $g$ will be said to satisfy the condition $\mathrm{IV}^{\prime}$ if at each point 3 on $g$ the inequality (4:1) holds subject to the conditions $(4: 2)$ and if furthermore the matrix

$$
\left\|\zeta_{i}, u_{i k}-\eta_{i j} v_{i k}\right\|
$$

of the coefficients in the bilinear form $(4: 1)$ has rank $n-p$ on $g$, where $p$ is the order of anormality of $g$. The matrix $(7: 1)$ has rank $n-p$ on $g$ if and 
only if the equations $(3: 4),(3: 6),(3: 7)$ have no solution $(\eta, \zeta, w)$ other than those described in Lemma $3: 1$. In the fixed end point case the matrix $(7: 1)$ has rank $n-p$ if and only if the end points of $g$ are not conjugate to each other, as is readily verified.

LEMMA 7:1. If in the separated end point case the extremal $g$ is non-singular and satisfies the condition $\mathrm{IV}^{\prime}$ and if the secondary end conditions are regular on $g$, then there exists for $g$ a conjugate system $U_{i k}, V_{i k}$ of secondary extremals whose determinant $\left|U_{i k}(x)\right|$ is different from zero on the interval $x^{1} \leqq x \leqq x^{2}$ determined by the end points 1 and 2 of $g$. Moreover the inequalities

$$
\begin{array}{rr}
b_{\mu \nu}^{1} z_{\mu} z_{\nu}-U_{i k}\left(x^{1}\right) V_{i j}\left(x^{1}\right) a_{j} a_{k}>0 & (\mu, \nu=1, \cdots, \rho ; i, j, k=1, \cdots, n), \\
-b_{\sigma \tau}^{2} z_{\sigma} z_{\tau}+U_{i k}\left(x^{2}\right) V_{i j}\left(x^{2}\right) b_{j} b_{k}>0 & (\sigma, \tau=\rho+1, \cdots, r)
\end{array}
$$

hold for every set of constants $\left(a_{k}, z_{\mu}\right) \neq(0,0)$ and $\left(b_{k}, z_{\sigma}\right) \neq(0,0)$ satisfying the equations

$$
U_{i k}\left(x^{1}\right) a_{k}=c_{i \mu}^{1} z_{\mu}, U_{i k}\left(x^{2}\right) b_{k}=c_{i \sigma}^{2} z_{\sigma} .
$$

For, if $p$ is the order of anormality of $g$, then by virtue of Lemma 3:1 we can select the first $p$ solutions of the sets $(3: 8)$ and $(3: 9)$ so that on $x^{1} x^{2}$ we have

$$
\eta_{i \gamma}(x) \equiv u_{i \gamma}(x) \equiv 0, \zeta_{i \gamma}(x) \equiv v_{i \gamma}(x) \quad(\gamma=1, \cdots, p)
$$

and so that the columns of the matrix $\left\|\zeta_{i \gamma}\left(x^{1}\right)\right\|$ are normed and orthogonalized. We then select the remaining solutions of these sets so that the relations

$$
\begin{aligned}
\zeta_{i \gamma}\left(x^{1}\right) \zeta_{i \alpha}\left(x^{1}\right)=0, \zeta_{i \gamma}\left(x^{1}\right) v_{i \alpha}\left(x^{1}\right) & =v_{i \gamma}\left(x^{1}\right) v_{i \alpha}\left(x^{1}\right)=0, \\
\zeta_{i \alpha} u_{i \beta}-\eta_{i \alpha} v_{i \beta} & =\delta_{\alpha \beta} \quad(\alpha, \beta=p+1, \cdots, n)
\end{aligned}
$$

hold, where $\delta_{\alpha \beta}$ is the Kronecker delta. In order to obtain the relations ( $\left.7: 6\right)$ we note that, since the conjugate systems $\eta_{i k}, \zeta_{i k}$ and $u_{i k}, v_{i k}$ have the secondary extremals $(7: 4)$ in common, it follows that

$$
\zeta_{i \gamma} u_{i k}-\eta_{i \gamma} v_{i k}=0, \zeta_{i k} u_{i \gamma}-\eta_{i k} v_{i \gamma}=0 \quad(\gamma=1, \cdots, p) .
$$

The determinant

$$
\left|\zeta_{i \alpha} u_{i \beta}-\eta_{i \alpha} v_{i \beta}\right| \quad(\alpha, \beta=p+1, \cdots, n)
$$

must therefore be different from zero if the matrix $(7: 1)$ is to have rank $n-p$. The relations $(7: 6)$ are now obtained by replacing the solutions $\eta_{i \alpha}, \zeta_{i \alpha}, w_{\mu \alpha}$ by the solutions $\eta_{i \alpha} A_{\alpha \beta}, \zeta_{i \alpha} A_{\alpha \beta}, w_{\mu \alpha} A_{\alpha \beta}$, where the matrix $\left\|A_{\alpha \beta}\right\|$ is the reciprocal of the matrix $(7: 8)$.

The secondary extremals $U_{i k}, V_{i k}$ taking the initial values 


$$
\begin{array}{ll}
U_{i \gamma}\left(x^{1}\right)=\zeta_{i \gamma}\left(x^{1}\right), & U_{i \alpha}\left(x^{1}\right)=\eta_{i \alpha}\left(x^{1}\right)+u_{i \alpha}\left(x^{1}\right) \quad(\gamma=1, \cdots, p), \\
V_{i \gamma}\left(x^{1}\right)=0, & V_{i \alpha}\left(x^{1}\right)=\zeta_{i \alpha}\left(x^{1}\right)+v_{i \alpha}\left(x^{1}\right)(\alpha=p+1, \cdots, n)
\end{array}
$$

can be shown to have the properties described in the theorem. In the first place these secondary extremals are mutually conjugate, as is easily seen, with the help of equations $(7: 5),(7: 6),(7: 9)$ and the conjugacy of the systems $\eta_{i k}, \zeta_{i k}$ and $u_{i k}, v_{i k}$. Moreover the determinant $\left|U_{i k}(x)\right|$ is different from zero on $x^{1} x^{2}$. In order to prove this we use the relations

$$
\zeta_{i \gamma} U_{i \epsilon}=\delta_{\gamma \epsilon}, \zeta_{i \gamma} U_{i \alpha}=0 \quad(\gamma, \epsilon=1, \cdots, p ; \alpha=p+1, \cdots, n)
$$

which hold identically on $x^{1} x^{2}$ by virtue of Lemma $3: 3$ and the equations $(7: 4),(7: 7),(7: 9)$ together with the fact that the columns of the matrix $\left\|\zeta_{i \gamma}\left(x^{1}\right)\right\|$ are normed and orthogonalized. If now the determinant $\left|U_{i k}(x)\right|$ were zero at a point $x^{3}$ on $x^{1} x^{2}$ then there would exist constants $c_{k}$ not all zero such that $U_{i k}\left(x^{3}\right) c_{k}=0$. By multiplying these equations by $\zeta_{i \gamma}\left(x^{3}\right)$, adding, and using equations $(7: 10)$ it would follow that the constants $c_{1}, \cdots, c_{p}$ would all be zero and hence that

$$
U_{i \alpha}\left(x^{3}\right) c_{\alpha}=\eta_{i \alpha}\left(x^{3}\right) c_{\alpha}+u_{i \alpha}\left(x^{3}\right) c_{\alpha}=0 \quad(\alpha=p+1, \cdots, n) .
$$

The equations (4:2) would then be satisfied by the set $a_{k}=c_{k}, b_{k}=-c_{k}$ and for these constants the bilinear form (4:1) would take the value $a_{\alpha} b_{\alpha}=-c_{\alpha} c_{\alpha}$ $<0$ by virtue of the equations $(7: 6)$ and $(7: 7)$. But this would contradict the condition IV'. The determinant $\left|U_{i k}\right|$ must therefore be different from zero on $x^{1} x^{2}$.

We shall now establish the first of the inequalities $(7: 2)$. In order to do this we first note that the constants $a_{1}, \cdots, a_{p}$ in equations $(7: 3)$ are all zero, as can be easily seen, by multiplying the first $n$ of these equations by $\zeta_{\text {ir }}\left(x^{1}\right)$, adding, and applying the equations $(7: 10)$ and the analogue of equations $(3: 11)$ for the separated end point case. We use the abbreviations

$$
\begin{aligned}
& \eta_{i}=\eta_{i \alpha} a_{\alpha}, \zeta_{i}=\zeta_{i \alpha} a_{\alpha}, \quad w_{\mu}=w_{\mu \alpha} a_{\alpha}, \\
& u_{i}=u_{i \alpha} a_{\alpha}, v_{i}=v_{i \alpha} a_{\alpha}, \quad z_{\mu}=w_{\mu}+w_{\mu}^{\prime} \quad(\alpha=p+1, \cdots, n)
\end{aligned}
$$

and find that the set $\eta_{i}, \zeta_{i}, w_{\mu}$ satisfies the equations (3:6). Moreover by the use of equations $(3: 6)$ and $(7: 3)$ it follows readily that at $x=x^{1}$

$$
\begin{aligned}
U_{i k} a_{k}-c_{i \mu}^{1} z_{\mu} & =u_{i}-c_{i \mu}^{1} w_{\mu}^{\prime}=0 \\
b_{\mu \nu}^{1} w_{\nu} w_{\mu}^{\prime} & =\zeta_{i} c_{i \mu}^{1} w_{\mu}^{\prime}=\zeta_{i} u_{i}, \\
b_{\mu \nu} w_{\nu} w_{\mu} & =\zeta_{i} c_{i \mu}^{1} w_{\mu}=\zeta_{i} \eta_{i} .
\end{aligned}
$$

With the help of the last two formulas and the equations $(7: 11)$ it is found that the first member of the relations $(7: 2)$ is expressible in the form 


$$
\begin{aligned}
b_{\mu \nu}^{1}\left(w_{\mu}+w_{\mu}^{\prime}\right)\left(w_{\nu}+w_{\nu}^{\prime}\right)-\left(\eta_{i}+u_{i}\right)\left(\zeta_{i}+\right. & \left.v_{i}\right) \\
& =\left[b_{\mu \nu}^{1} w_{\mu}^{\prime} w_{\nu}^{\prime}-u_{i} v_{i}\right]+\left[\zeta_{i} u_{i}-\eta_{i} v_{i}\right] .
\end{aligned}
$$

The second bracket is equal to the sum $a_{\alpha} a_{\alpha}$ by virtue of equations $(7: 6)$ and ( $7: 11)$ and is positive unless the constants $a_{\alpha}$ are all zero, in which case the constants $z_{\mu}$ in equations $(7: 3)$ are also all zero since the secondary end conditions are regular. The first bracket in the last equation is positive or zero. For, as a consequence of the regularity of the secondary end conditions there exists for every set of constants $w_{\mu}^{\prime}$ a secondary extremal $\eta_{i 0}, \zeta_{i 0}$ satisfying the conditions (3:6) with $w_{\mu}=w_{\mu}^{\prime}$. The set $\eta_{i 0}, \zeta_{i 0}, w_{\mu}^{\prime}$ is expressible linearly with constants $c_{k}$ in terms of the set $(3: 8)$. Moreover it is clear that $\eta_{i 0}\left(x^{1}\right)=u_{i}\left(x^{1}\right)$. Hence we have at $x=x^{1}$

$$
\begin{aligned}
b_{\mu \nu}^{1} w_{\mu}^{\prime} w_{\nu}^{\prime}-u_{i} v_{i} & =\zeta_{i 0} \eta_{i 0}-u_{i} v_{i} \\
& =\zeta_{i 0} u_{i}-\eta_{i 0} v_{i}=\left(\zeta_{i j} u_{i k}-\eta_{i j} v_{i k}\right) c_{j} a_{k}
\end{aligned}
$$

and this expression must be positive or zero by IV'. This proves the first inequality $(7: 2)$. The second can be established by the same method. The proof of Lemma $7: 1$ is now complete

We also have the further useful lemma:

LEMMA 7:2. If the extremal $g$ is non-singular and its end points are not conjugate to each other, then the end points of every differentiably admissible arc $g_{2}$ for the accessory minimum problem can be joined by a secondary extremal.

To prove this let $q$ be the order of anormality of $g$ on $x^{1} x^{2}$ and suppose that the first $q$ secondary extremals $u_{i \gamma}, v_{i \gamma}(\gamma=1, \cdots, q)$ of the set $u_{i j}, v_{i j}$ $(j=1, \cdots, 2 n)$ appearing in Theorem 5:1 have been chosen so that $u_{i \gamma} \equiv 0$ on $x^{1} x^{2}$. Since the end points of $g$ are not conjugate, the end values of the remaining $2 n-q$ secondary extremals of this set form a set of $2 n-q$ linearly independent solutions of the equations

$$
v_{i \gamma}\left(x^{2}\right) \eta_{i}{ }^{2}=v_{i \gamma}\left(x^{1}\right) \eta_{i}{ }^{1} \quad(\gamma=1, \cdots, q),
$$

by Lemma $3: 3$, and every solution $\eta_{i}{ }^{1}, \eta_{i}{ }^{2}$ of these equations is expressible linearly in terms of these $2 n-q$ solutions. The end points of $g_{2}$ satisfy these equations, by Lemma $3: 3$. This proves Lemma $7: 2$.

By the Clebsch condition III' is meant the conditions of Theorem 4:5 with

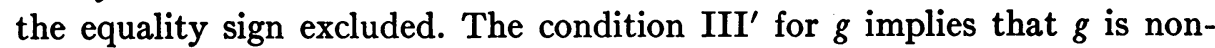
singular (V, p. 735). We can now prove the following lemma: 


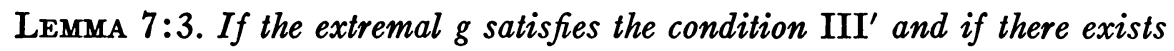
for $g$ a conjugate system $U_{i k}, V_{i k}$ of secondary extremals whose determinant $\left|U_{i k}(x)\right|$ is different from zero on $x^{1} x^{2}$, then every secondary extremal $u_{i}, v_{i}$ is an extremal of a Mayer field defined over the region $\mathfrak{F}_{\Omega}$ of points $(x, \eta)$ whose $x$ projections lie on the interval $x^{1} x^{2}$. Moreover the analogue of the condition (6:4) holds in $\mathfrak{r}_{\Omega}$.

For, the $n$-parameter family of secondary extremals

$$
\eta_{i}=u_{i}+U_{i k} a_{k}, \zeta_{i}=v_{i}+V_{i k} a_{k}
$$

contains the extremal $u_{i}, v_{i}$ for values $(a)=(0)$ and simply covers the region $\mathfrak{F}_{\Omega}$. Moreover the Hilbert integral $I^{*}$ formed for the function $2 \Omega$ is independent of the path on the hyperplane $x=x^{2}$. The family $(7: 12)$ therefore defines a field over $\mathfrak{F}_{\Omega}(\mathrm{V}$, p. 733; VII, p. 571). The last statement in the lemma follows at once from the condition III' by the use of Taylor's expansion.

8. Necessary and sufficient conditions for the second variation to be positive definite. The second variation $J_{2}$ is said to be positive definite along the extremal $g$ if the inequality $J_{2}(\eta, w)>0$ is true for every set of admissible variations $(\eta, w) \not \equiv(0,0)$ belonging to $g$.

THEOREM 8:1. If in the separated end point case the extremal $g$ is nonsingular and the secondary end conditions are regular on $g$, then the second variation $J_{2}(\eta, w)$ is positive definite along $g$ if and only if the conditions III' and $^{\prime}$ IV' hold along $\mathrm{g}$.

The necessity of the conditions III' and IV $^{\prime}$ follows at once from Theorems $4: 1,4: 5$ and the remarks preceding Lemma $7: 1$. The sufficiency of these conditions follows readily from Lemmas $7: 1,7: 3$ and Theorem 6:1 applied to the secondary extremal $u_{i} \equiv v_{i} \equiv 0$, the conditions $(7: 2)$ and $(7: 3)$ implying the positive definiteness of the analogue of the quadratic form $(6: 3)$.

We now turn to the case in which the end conditions are of the form $(1: 3)$. By the condition $\mathrm{V}^{\prime}$ is meant the necessary conditions of Theorem $4: 2$ with the added assumption that the equation $Q(z)=0$ holds only in case $\eta_{i \rho} z_{\rho} \equiv 0$, $w_{h \rho} z_{\rho}=0$ on $x^{1} x^{2}$. The condition $\mathrm{V}^{\prime}$ for $g$ prevents its end points from being conjugate to each other. For if the end points 1 and 2 of $g$ were conjugate then there would exist a secondary extremal $\eta_{i}, \mu_{\beta}$ with $\eta_{i}\left(x^{1}\right)=\eta_{i}\left(x^{2}\right)=0$ and $(\eta) \not \equiv(0)$ on $x^{1} x^{2}$. The set $\eta_{i}, \mu_{\beta}, w_{h}=0$ would then be expressible linearly with constants $z_{\rho}$ in terms of the set $\eta_{i \rho}, \mu_{\beta \rho}, w_{h \rho}$ appearing in the definition of the quadratic form (4:5). For these values of $(z)$ we would have $Q(z)=0$, as is easily seen, with the help of the formula (4:4) and the usual integration by 
parts. It follows that the end points of $g$ cannot be conjugate if the condition $\mathrm{V}^{\prime}$ is to hold along $g$.

TheOREm 8:2. If the extremal $g$ is non-singular then the second variation $J_{2}(\eta, w)$ is positive definite along $g$ if and only if the conditions $\mathrm{III}^{\prime}$ and $\mathrm{V}^{\prime}$ hold along $g$.

It is clear that the conditions $\mathrm{III}^{\prime}, \mathrm{V}^{\prime}$ are necessary. In order to show that they are sufficient we note first that the condition $V^{\prime}$ for $g$ implies the condition IV' for the fixed end point case. Lemma 7:1 now tells us that there exists a conjugate system $U_{i k}, V_{i k}$ of secondary extremals whose determinant $\left|U_{i k}(x)\right|$ is different from zero on $x^{1} x^{2}$. From Lemma 7:3 and Theorem 6:1 we conclude that every secondary extremal $u_{i}, v_{i}$ affords a proper minimum to the integral

$$
I_{2}=\int_{x^{1}}^{x^{2}} 2 \omega\left(x, \eta, \eta^{\prime}\right) d x
$$

relative to differentiably admissible $\operatorname{arcs} \eta_{i}(x)$ joining its end points.

Suppose now that $\eta_{j}, w_{h}$ is an admissible arc for the accessory minimum problem. By Lemma $7: 2$ there exists a secondary extremal $u_{i}, v_{i}$ joining its end points. We have accordingly

$$
J_{2}(\eta, w)-J_{2}(u, w)=I_{2}(\eta)-I_{2}(u) \geqq 0,
$$

the equality being valid only in case $(\eta) \equiv{ }^{\prime}(u)$. From the definition of the quadratic form $Q(z)$ it is clear that there exist constants $z_{\rho}$ such that $Q(z)=J_{2}(u, w)$. From the condition $\mathrm{V}^{\prime}$ and the relation (8:1) we now conclude that $J_{2}(\eta, w)>0$ unless $(\eta, w) \equiv(0,0)$, as was to be proved.

The extremal $g$ will be said to satisfy the condition VI' $^{\prime}$ if the quadratic form (4:5) is positive on $g$ and vanishes only in case $\eta_{i_{\rho}} z_{\rho} \equiv 0, w_{h \rho} z_{\rho}=0$ on $x^{1} x^{2}$, and if furthermore there is no point 3 conjugate to the initial point 1 on $g$. We can now prove the following theorem:

THEOREM 8:3. If the extremal $g$ is non-singular and the order $q$ of anormality of $g$ is the same on every sub-interval $x^{1} x^{3}$ of $x^{1} x^{2}$, then the second variation $J_{2}(\eta, w)$ is positive definite along $g$ if and only if the conditions $\mathrm{III}^{\prime}, \mathrm{VI}^{\prime}$ hold along $g$.

The proof of this theorem is like that of Theorem 8:2 provided that we can show that there exists for $g$ a conjugate system $U_{i k}, V_{i k}$ of secondary extremals having its determinant $\left|U_{i k}(x)\right|$ different from zero on $x^{1} x^{2}$. This latter result will be obtained by a method first used by Morse (VII, pp. 574-6) for the problem of Lagrange and later adapted to the problem of 
Mayer by Bliss and Hestenes (XVII, pp. 320-2). In the proof we suppose that the conjugate system $\eta_{i k}, \zeta_{i k}$ of Theorem 5:2 has been chosen to take the values $\delta_{i k}, B_{i k}$ at $x=x^{2}$, where $\delta_{i k}$ is the Kronecker delta and $B_{i k}=B_{k i}$. "Lemma 8:2" of Bliss and Hestenes now holds as before. Similarly "Lemma $8: 3$ " is true, as is easily seen with the help of the following remarks. Although a secondary extremal $\eta_{i}, \zeta_{i}$ joining the points $(x, \eta)=\left(x^{1}, 0\right)$ and $(x, \eta)=\left(x^{2}, a\right)$ is not necessarily an extremal of the field it has associated with it a secondary extremal $\eta_{i}-c_{\gamma} u_{i \gamma}, \zeta_{i}-c_{\gamma} v_{i \gamma}(\gamma=1, \cdots, q)$ belonging to the field, where $q$ is the order of anormality of $g$ on $x^{1} x^{2}$ and $u_{i \gamma}, v_{i \gamma}$ are $q$ linearly independent secondary extremals having $u_{i \gamma} \equiv 0$ on $x^{1} x^{2}$. Moreover the values of the integral " $I_{2}$ " along these two extremals are the same. The remainder of the proof is now like that of "Theorem 8:1" of Bliss and Hestenes.

We now turn to the accessory boundary value problem. Its characteristic roots are all real (XIV, p. 774; XIX, p. 394). We have the following theorem:

THEOREM 8:4. If the extremal $g$ is non-singular and the secondary end conditions are regular on $g$, then the second variation $J_{2}(\eta, w)$ is positive definite (positive) along $g$ if and only if the condition III' holds along $g$ and the characteristic roots of the accessory boundary value problem are all positive (non-negative).

According to the remarks preceding Lemma 3:4 we may suppose that $g$ is normal. The theorem then follows from a result given by Hu (XIX, p. 413).

The theorem can also be established with the help of the condition $\mathrm{V}^{\prime}$. A method will be outlined briefly as follows. We first replace the integrand $2 \omega$ in the functional $J_{2}(\eta, w)$ by $2 \omega-\sigma \eta_{i} \eta_{i}$ and obtain a functional $J_{2}(\eta, w, \sigma)$. The preceding theorems concerning the functional $J_{2}(\eta, w)$ are valid also for the functional $J_{2}(\eta, w, \sigma)$ when the obvious changes due to the introduction of the parameter $\sigma$ are made. By an argument like that given by Morse (VIII, pp. 533-4) it is found that for $\sigma$ sufficiently large and negative the functional $J_{2}(\eta, w, \sigma)$ will be positive definite relative to sets of admissible variations $(\eta, w) \not \equiv(0,0)$. Let $\sigma_{0}$ be the least upper bound of the values of $\sigma$ for which $J_{2}(\eta, w, \sigma)$ is positive definite. It will be shown below that $\sigma_{0}$ must be finite. We shall now show that $\sigma_{0}$ is a characteristic root. The functional $J_{2}\left(\eta, w, \sigma_{0}\right)$ must be positive since otherwise there would exist an admissible $\operatorname{arc} \eta_{i}, w_{h}$ such that $J_{2}(\eta, w, \sigma)<0$ for $\sigma=\sigma_{0}$ and hence for $\sigma<\sigma_{0}$ and suffciently near to $\sigma_{0}$, which is not the case. If the functional $J_{2}\left(\eta, w, \sigma_{0}\right)$ were positive definite then by Theorem 8:2 the condition $V^{\prime}$ would hold for this functional. By the use of Lemma 3:2 applied to sub-intervals of the form $x^{1} x^{3}$ and $x^{3} x^{2}$ and with the help of well known theorems on quadratic forms it could then be shown that the condition $\mathrm{V}^{\prime}$ would hold for the functional $J_{2}(\eta, w, \sigma)$ for values of $\sigma$ slightly larger than $\sigma_{0}$. The functional $J_{2}(\eta, w, \sigma)$ would then be positive definite for these values of $\sigma$, by Theorem $8: 2$, and $\sigma_{0}$ 
could not be the least upper bound for such values of $\sigma$. It follows that there exists at least one admissible arc $\eta_{i}, w_{h}$ with $(\eta) \not \equiv(0)$ on $x^{1} x^{2}$ such that $J_{2}\left(\eta, w, \sigma_{0}\right)=0$. As in the proof of Lemma 3:4 it is seen that this arc has associated with it a set of multipliers $\mu_{0}=1, \mu_{\beta}(x)$ such that the functions $\eta_{i}, \mu_{\beta}$ define a secondary extremal for the problem of minimizing the functional $J_{2}\left(\eta, w, \sigma_{0}\right)$ in the class of admissible variations $\eta_{i}, w_{h}$ belonging to $g$. The functions $\eta_{i}, \mu_{\beta}$ therefore form a characteristic solution and $\sigma_{0}$ a characteristic root.

In order to show that $\sigma_{0}$ is finite we note that there exists at least one set of admissible variations $\eta_{i}, w_{h}$ having $(\eta) \not \equiv(0)$ on $x^{1} x^{2}$ since the accessory minimum problem can be made normal. For this set the functional $J_{2}(\eta, w, \sigma)$ can be made negative by taking $\sigma$ sufficiently large and positive. Consequently $\sigma_{0}$ must be finite. This proves the theorem.

9. Sufficient conditions for relative minima. The end conditions $(1: 3)$ are said to be regular on the admissible arc $g$ under consideration if the matrix of the derivatives of the functions $x^{s}(\alpha), y_{i}{ }^{s}(\alpha)$ has rank $r$ for $(\alpha)=(0)$. The arc $g$ is said to satisfy the non-tangency condition if the manifold $y_{i}{ }^{s}=y_{i}\left(x^{s}\right)$ $(s=1,2)$ and the terminal manifold $x^{s}=x^{s}(\alpha), y_{i}{ }^{s}=y_{i}{ }^{s}(\alpha)$ possess no common tangent line at the point $(\alpha)=(0)$ on the terminal manifold. The end conditions are regular and the non-tangency condition holds on $g$ if and only if the secondary end conditions (3:2) are regular on $g$ (VIII, pp. 525-6). No generality is lost in assuming that the end conditions are regular and the nontangency condition holds on $g$, as can be seen from the proof of Theorem 9:2 below.

The symbol I will be used to denote the necessary condition of Theorem $2: 1$. An admissible arc $g$ with a set of multipliers $\lambda_{0}, \lambda_{\beta}(x)$ is said to satisfy the Weierstrass condition $\mathrm{II}^{\prime} \mathfrak{R}$ if at each element $\left(x, y, y^{\prime}, \lambda\right)$ in a neighborhood $\mathfrak{R}$ of those on $g$ the inequality

$$
E\left[x, y, y^{\prime}, \lambda, Y^{\prime}\right]>0
$$

holds for every admissible set $\left(x, y, Y^{\prime}\right) \neq\left(x, y, y^{\prime}\right)$. The Clebsch condition III' and the conditions IV', V', VI' have been described in $\$ \S 7$ and 8. The last three conditions can readily be expressed in terms of the extremal family in a manner analogous to that given by Bliss (IX, pp. 265-6; cf. XVIII, p. 483).

THEOREM 9:1. Let $g$ be an admissible arc for the problem of Bolza with separated end conditions. Suppose that the end conditions (1:4) are regular and that the non-tangency condition holds on $g$. If $g$ has no corners and satisfies the condiditions $\mathrm{I}, \mathrm{II}_{\mathfrak{R}^{\prime}}, \mathrm{III}^{\prime}, \mathrm{IV}^{\prime}$ with a set of multipliers $\lambda_{0}=1, \lambda_{\beta}(x)$, then $g$ affords a proper strong relative minimum to the functional $J$. 
From the conditions $I$ and III' we conclude that $g$ is a non-singular extremal since it has no corners $(V, p$. 735). The theorem will now be established by showing that the hypotheses of Theorem $6: 1$ are fulfilled.

As a first step we note that $g$ is a member for values $x^{1} \leqq x \leqq x^{2}, a_{i}=a_{i 0}$ $(i=1, \cdots, n)$ of an $n$-parameter family of extremals whose equations in the canonical variables $x, y_{i}, z_{i}=F_{y^{\prime}}$, are of the form

$$
y_{i}=y_{i}\left(x, a_{1}, \cdots, a_{n}\right), z_{i}=z_{i}\left(x, a_{1}, \cdots, a_{n}\right) .
$$

The functions $y_{i}, y_{i x}, z_{i}, z_{i x}$ have continuous first and second derivatives for all values $(x, a)$ in a neighborhood of those belonging to $g$. The parameters $(a)$ can be chosen so that along $g$

$$
y_{i a_{k}}\left(x, a_{0}\right) \equiv U_{i k}(x), z_{i a_{k}}\left(x, a_{0}\right) \equiv V_{i k}(x),
$$

where the functions $U_{i k}, V_{i k}$ are secondary extremals having the properties described in Lemma 7:1. Moreover the family of extremals (6:1) defines a Mayer field over a neighborhood $\mathfrak{F}$ of $g$. The proof of the existence of such a family is like that given by Bliss and Hestenes (XVII, pp. 322-3) and by Morse (VII, p. 576) with help of Lemma 7:1. Let $p_{i}(x, y), \lambda_{\beta}(x, y)$ be the slope functions and the multipliers of the field. It is clear that the field $\mathfrak{F}$ can be taken so small that the elements $[x, y, p(x, y), \lambda(x, y)]$ will lie in the neighborhood $\mathfrak{R}$ specified by the condition $\operatorname{II}_{\mathfrak{N}^{\prime}}$. The inequality $(6: 4)$ then holds at each point in $\mathfrak{F}$.

The identity $(6: 2)$ follows at once from the transversality condition $(2: 2)$. In order to show that the quadratic form (6:3) is positive definite on $g$ it is convenient to express $I^{*}$ in terms of the variables $x, a_{1}, \cdots, a_{n}$ instead of the variables $x, y_{1}, \cdots, y_{n}$. In doing so we replace the functions $p_{i}(x, y), \lambda_{\beta}(x, y)$ by the functions $y_{i x}(x, a), \lambda_{\beta}(x, a)$, where $\lambda_{\beta}(x, a)$ are the multipliers belonging to the family $(9: 1)$. We use the following abbreviations:

$$
\begin{array}{rlrl}
\delta y_{i} & =y_{i a_{k}} d a_{k}, \quad \delta z_{i} & =z_{i a_{k}} d a_{k}, & d y_{i}=y_{i x} d x+\delta y_{i}, \\
d y_{i}^{\prime} & =y_{i x x} d x+\delta y_{i}^{\prime}, d^{2} y_{i} & =y_{i x} d^{2} x+d y_{i}^{\prime} d x+\delta y_{i}^{\prime} d x+\delta^{2} y_{i} .
\end{array}
$$

With the help of the Euler-Lagrange equations (2:3) it is found that

$$
d\left(F_{y^{\prime}}\right)=F_{y_{i}} d x+\delta z_{i} .
$$

Hence at the initial point 1 on $g$ we have

$$
\begin{aligned}
-d I^{*}+d \theta^{1}= & -\left(F-y_{i}^{\prime} F_{y_{i^{\prime}}}\right) d x-F_{y_{i}^{\prime}} d y_{i}+d \theta^{1}, \\
-d^{2} I^{*}+d^{2} \theta^{1}= & -\left(F-y_{i}^{\prime} F_{y_{i}^{\prime}}\right) d^{2} x-F_{y^{\prime}} d^{2} y_{i}-\left(F_{x}-y_{i}^{\prime} F_{y_{i}}\right)(d x)^{2} \\
& -2 F_{y_{i}} d y_{i} d x-\delta z_{i} \delta y_{i}+d^{2} \theta^{1} .
\end{aligned}
$$


If now we replace the first and second differentials of $x$ and $y_{i}$ by their values in terms of the differentials of $\alpha_{\mu}$ it is found with the help of equations (3:5), (9:2), and Lemma $7: 1$ that the inequality

$$
-d^{2} I^{*}+d^{2} \theta^{1}=b_{\mu \nu}{ }^{1} d \alpha_{\mu} d \alpha_{\nu}-\delta y_{i} \delta z_{i}>0 \quad(\mu, \nu=1, \cdot \cdot, \rho)
$$

holds at the point 1 on $g$ for every set $\left(d a_{k}, d \alpha_{\mu}\right) \neq(0,0)$ satisfying the conditions $\delta y_{i}\left(x^{1}\right)=c_{i \mu}^{1} d \alpha_{\mu}$. By a similar argument it can be shown that at the final end point 2 on $g$ the inequality

$$
d^{2} I^{*}-d^{2} \theta^{2}=-b_{\sigma \tau}{ }^{2} d \alpha_{\sigma} d \alpha_{\tau}+\delta y_{i} \delta z_{i}>0 \quad(\sigma, \tau=\rho+1, \cdots, r)
$$

holds for every set $\left(d a_{k}, d \alpha_{\sigma}\right) \neq(0,0)$ satisfying the equations $\delta y_{i}\left(x^{2}\right)=c_{i \sigma}{ }^{2} d \alpha_{\sigma}$. The last two inequalities show that the quadratic form (6:3) is positive definite on $g$. Theorem $6: 1$ now justifies the theorem that was to be proved.

Theorem 9:1 will now be used in order to obtain sufficient conditions for the problem of Bolza with end conditions of the type (1:3). In the following theorem it should be noted that the assumptions of regularity of the end conditions and of non-tangency are not needed.

THEOREM 9:2. If an admissible arc $g$ without corners satisfies the conditions I, II $\mathfrak{R}^{\prime}, \mathrm{III}^{\prime}$ with a set of multipliers $\lambda_{0}=1, \lambda_{\beta}(x)$ and if the second variation $J_{2}(\eta, w)$ is positive definite along $g$, then $g$ affords a proper strong relative minimum to the functional $J$.

In order to prove the theorem we note first that a problem of Bolza with end conditions of the form $(1: 3)$ is equivalent to the problem of finding in the class of arcs

$$
y_{j}=y_{j}(x) \quad\left(x^{1} \leqq x \leqq x^{2} ; j=1, \cdots, n+r\right)
$$

and sets $\alpha_{h}, \gamma_{h}(h=1, \cdots, r)$ satisfying the differential equations and end conditions

$$
\begin{array}{rlrl}
\phi_{\beta}\left(x, y_{i}, y_{i}^{\prime}\right) & =0, & y_{n+h}^{\prime}=0 & (i=1, \cdots, n ; h=1, \cdots, r), \\
x^{1}=x^{1}(\alpha), & y_{i}{ }^{1}=y_{i}{ }^{1}(\alpha), & y_{n+h^{1}}=\alpha_{h}, \\
x^{2}=x^{2}(\gamma), & y_{i}{ }^{2}=y_{i}{ }^{2}(\gamma), & y_{n+h^{2}}=\gamma_{h}
\end{array}
$$

one which minimizes the functional $J$. This equivalence follows from the fact that the functions $y_{n+h}(x)$ are all constants and hence take the values $\alpha_{h}=\gamma_{h}$ at $x=x^{1}$ and $x=x^{2}$. The new problem is a problem of Bolza with separated end conditions and will be called the transformed problem. Let $g_{1}$ be the admissible arc for the transformed problem which corresponds to the arc $g$ of the theorem. It is easily seen that the new end conditions are regular and that the non-tangency condition holds on $g_{1}$. The arc $g_{1}$ also satisfies the con- 
ditions I, III $\mathfrak{R}^{\prime}$, III' for the transformed problem with the set of multipliers $\lambda_{0}=1, \lambda_{\beta}(x), \lambda_{m+h}(x)$, where the multipliers $\lambda_{m+h}(x)$ are constants determined by the transversality condition $(2: 2)$. Moreover there is one-to-one correspondence between the admissible variations for the two problems and along corresponding admissible variations the values of the second variation for the two problems are the same. Theorems 8:1 and 9:1 therefore tell us that $g_{1}$ furnishes a proper strong relative minimum for the transformed problem and hence that $g$ furnishes a proper strong relative minimum for the original problem, as was to be proved.

From Theorems 8:2 and 9:2 we obtain the following result:

THEOREM 9:3. If an admissible arc $g$ without corners satisfies the conditions $\mathrm{I}^{\prime}, \mathrm{II}_{\mathfrak{N}^{\prime}}, \mathrm{III}^{\prime}, \mathrm{V}^{\prime}$ with a set of multipliers $\lambda_{0}=1, \lambda_{\beta}(x)$, then $\mathrm{g}$ affords a proper strong relative minimum to the functional $J$.

Combining Theorems 8:3 and 9:2 we obtain the further

THEOREM 9:4. The results of Theorem 9:3 remain valid when the condition $\mathrm{V}^{\prime}$ is replaced by the condition $\mathrm{VI}^{\prime}$ provided that the order $q$ of anormality of $g$ is the same on every sub-interval $x^{1} x^{3}$ of the interval $x^{1} x^{2}$ determined by the end points of $g$.

The last two theorems are extensions of the sufficient conditions given by Bliss (IX, p. 271). The following theorem gives an extension of the sufficient conditions given by Morse (VIII, p. 528) and $\mathrm{Hu}$ (XIX, p. 417) and is obtained by combining Theorems $8: 4$ and $9: 2$.

THEOREM 9:5. Suppose that the end conditions are regular and that the nontangency condition holds on an admissible arc $g$ having no corners. If $g$ satisfies the conditions $\mathrm{I}, \mathrm{II}_{\mathfrak{N}^{\prime}}, \mathrm{III}^{\prime}$ with a set of multipliers $\lambda_{0}=1, \lambda_{\beta}(x)$ and if the characteristic roots of the accessory boundary value problem are all positive, then $g$ af: fords a proper strong relative minimum to the functional $J$.

Sufficient conditions for a weak relative minimum can be obtained in the usual manner by omitting the condition $\mathrm{II}_{\mathfrak{N}}{ }^{\prime}$ in the above theorems $(\mathrm{V}, \mathrm{pp}$. 736-7).

The following example shows clearly that the sufficient conditions here given actually do not imply the normality relations which we have proposed to exclude. Let $h$ be a small positive constant and let $A(x), B(x)$ be functions satisfying the conditions

$$
\begin{aligned}
& A(x)>0 \text { on } x^{1} \leqq x<x^{1}+h, \quad A(x) \equiv 0 \text { on } x^{1}+h \leqq x \leqq x^{2} \\
& B(x) \equiv 0 \text { on } x^{1} \leqq x \leqq x^{2}-h, \quad B(x)>0 \text { on } x^{2}-h<x \leqq x^{2}
\end{aligned}
$$


and having continuous derivatives of the first three orders. The segment $g$ of the $x$-axis between $x^{1}$ and $x^{2}$ then furnishes a proper strong minimum to the integral

$$
J=\int_{x^{1}}^{x^{2}}\left(1+y_{1}^{\prime 2}\right)^{1 / 2} d x
$$

in the class of arcs $(1: 1)$ with $n=4$ satisfying the differential equations

$$
y_{2}^{\prime}=y_{1}^{\prime}+A(x) y_{1}, \quad y_{3}^{\prime}=y_{1}^{\prime}+B(x) y_{1}, \quad y_{4}^{\prime}=0
$$

and joining the two fixed points $(x, y)=\left(x^{1}, 0\right)$ and $(x, y)=\left(x^{2}, 0\right)$. The order $p$ of anormality of $g$ is readily found to be unity. The order $q$ of anormality of $g$ is unity on every sub-interval $x^{\prime} x^{\prime \prime}$ satisfying the conditions $x^{1} \leqq x^{\prime}<x^{1}+h$, $x^{2}-h<x^{\prime \prime} \leqq x^{2}$. If one of these conditions holds, then $q=2$. If neither holds, then $q=3$. It follows that the sufficient conditions given heretofore are not applicable to $g$. However $g$ satisfies the sufficient conditions here given with the set of multipliers $\lambda_{0}=1, \lambda_{\beta}(x) \equiv 0$, except for those in Theorem 9:4.

I. Hadamard, Leçons sur le Calcul des Variations, 1910.

II. Bolza, Über den "anormalen Fall" beim Lagrangeschen und Mayerschen Problem mit gemischten Bedingungen und variablen Endpunkten, Mathematische Annalen, vol. 74 (1913), pp. 430-446.

III. Bliss, The problem of Mayer with variable end points, these Transactions, vol. 19 (1918), pp. 305-314.

IV. Bliss, $A$ boundary value problem of the calculus of variations, Bulletin of the American Mathematical Society, vol. 32 (1926), pp. 317-331.

V. Bliss, The problem of Lagrange in the calculus of variations, American Journal of Mathematics vol. 32 (1930), pp. 673-744.

VI. Morse and Myers, The problems of Lagrange and Mayer with variable end points, Proceedings of the American Academy of Arts and Sciences, vol. 66 (1931), pp. 235-253.

VII. Morse, Sufficient conditions in the problem of Lagrange with fixed end points, Annals of Mathematics, (2), vol. 32 (1931), pp. 567-577.

VIII. Morse, Sufficient conditions in the problem of Lagrange with variable end points, American Journal of Mathematics, vol. 53 (1931), pp. 517-596.

IX. Bliss, The problem of Bolza in the calculus of variations, Annals of Mathematics, (2), vol. 33 (1932), pp. 261-274.

X. Bliss and Schoenberg, On the derivation of necessary conditions for the problem of Bolza, Bulletin of the American Mathematical Society, vol. 38 (1932), pp. 858-864.

XI. Myers, Adjoint systems in the problem of Mayer under general end conditions, Bulletin of the American Mathematical Society, vol. 38 (1932), pp. 303-312.

XII. Currier, The variable end point of the calculus of variations including a generalization of the classical Jacobi conditions, these Transactions, vol. 34 (1932), pp. 689-704.

XIII. Graves, On the Weierstrass condition for the problem of Bolza in the calculus of variations, Annals of Mathematics, (2), vol. 33 (1932), pp. 747-752.

XIV. Reid, $A$ boundary value problem associated with the calculus of variations, American Journal of Mathematics, vol. 54 (1932), pp. 769-790.

XV. Carathéodory, Die Theorie der zweiten Variation beim Problem von Lagrange, Sitzungsberichte der Bayerischen Akademie der Wissenschaften, 1932, pp. 99-114.

XVI. Carathéodory, Ueber die Einteilung der Variationsprobleme von Lagrange nach Klassen, Commentarii Mathematici Helvetici, vol. 5 (1933), pp. 1-10. 
XVII. Bliss and Hestenes, Sufficient conditions for a problem of Mayer in the calculus of variations, these Transactions, vol. 35 (1933), pp. 305-326; Contributions to the Calculus of Variations 193132, The University of Chicago Press, pp. 295-337.

XVIII. Hestenes, Sufficient conditions for the general problem of Mayer with variable end points, these Transactions, vol. 35 (1933), pp. 479-490; Contributions to the Calculus of Variations 1931-32, The University of Chicago Press, pp. 339-360.

XIX. Hu, Problem of Bolza and its accessory boundary value problem, Contributions to the Calculus of Variations 1931-32, The University of Chicago Press, pp. 361-443.

$\mathrm{XX}$. Bower, The problem of Lagrange with finite side conditions, Dissertation, The University of Chicago, 1933.

HARVARD UNIVERSTTY,

Cambrimge, Mass. 